\section{Edited by:}

Gabriel A. Silva, University of California, San Diego, USA

Reviewed by:

David C. Spray, Albert Einstein College of Medicine, USA

Erik Ullian, University of California,

USA

* Correspondence:

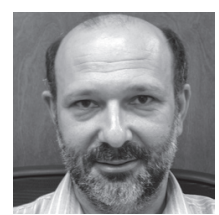

Vladimir Parpura, M.D., Ph.D., holds both a medical degree, awarded from the University of Zagreb in Croatia in 1989, and a doctorate, received in Neuroscience and Zoology from Iowa State University in 1993. He has held faculty appointments in the Department of Zoology and Genetics, Iowa State University, and the Department of Cell Biology and Neuroscience, University of California, Riverside. He is presently an Associate Professor in the Department of Neurobiology, University of Alabama, Birmingham. His research focuses on understanding the modulation of calcium-dependent glutamate release from astrocytes. vlad@uab.edu

\title{
Micropatterned substrates for studying astrocytes in culture
}

\section{William Lee and Vladimir Parpura*}

Department of Neurobiology, Center for Glial Biology in Medicine, Atomic Force Microscopy and Nanotechnology Laboratories, Civitan International Research Center, Evelyn F. McKnight Brain Institute, University of Alabama, Birmingham, AL, USA

Recent studies of the physiological roles of astrocytes have ignited renewed interest in the functional significance of these glial cells in the central nervous system. Many of the newly discovered astrocytic functions were initially demonstrated and characterized in cell culture systems. We discuss the use of microculture techniques and micropatterning of cell-adhesive substrates in studies of astrocytic $\mathrm{Ca}^{2+}$ excitability and bidirectional neuron-astrocyte signaling. This culturing approach aims to reduce the level of complexity of the system by limiting the interacting partners and by controlling the localization of cells. It provides tight control over experimental conditions allowing detailed characterization of cellular functions and intercellular communication. Although such a reductionist approach yields some difference in observations between astrocytic properties in culture and in situ, general phenomena discovered in cell culture systems, however, have also been found in vivo.

Keywords: astrocytes, bidirectional neuronal-glial interactions, $\mathrm{Ca}^{2+}$ dynamics, glutamate uptake and release, micropatterning

\section{PRIMER ON ASTROCYTIC FUNCTION AND APPROACHES TO STUDY THESE GLIAL CELLS} Astrocytes, a subtype of glial cells in the mammalian central nervous system (CNS), have long been regarded merely as structural and metabolic support for neurons. In the past twenty years, the advent of new techniques and tools in astrocytic research implicate additional regulatory roles that astrocytes provide in the CNS. Specifically, astrocytes can modulate synaptic transmission by the uptake of neurotransmitters (reviewed in Tzingounis and Wadiche, 2007) and/or by release of gliotransmitters (reviewed in Ni et al., 2007), as well as control blood flow (reviewed in Gordon et al., 2009). Also, it has become apparent that astrocytes play a role in pathological conditions, such as, epilepsy (reviewed in Binder and Steinhäuser, 2009), hepatic encephalopathy (reviewed in
Butterworth, 2009), Alexander (reviewed in Brenner et al., 2009) and Alzheimer diseases (Kuchibhotla et al., 2009).

Electrophysiology is arguably the most powerful and relevant technique to study neurons owing to the electrical excitability of these cells. However, the initial electrical recordings from astrocytes yielded rather unremarkable findings leading to classification of astrocytes as non-excitable cells. Unlike neurons, astrocytes do not encode information via generation of action potentials and display rather hyperpolarizing resting membrane potentials with little fluctuations. It should be noted, however, that electrical recordings from astrocytes have been instrumental in studying, for example, the activity of glutamate plasma membrane transporters on these cells in response to neuronal synaptic transmission (Mennerick and Zorumski, 1994). 


\section{Gliotransmitter}

Gliotransmitter is a chemical released from glial cells classified based on a working set of criteria: (i) synthesis by and/or storage in glia; (ii) regulated release triggered by physiological and/or pathological stimuli; (iii) activation of rapid ( $m s$ to $s$ ) responses in neighboring cells; (iv) a role in (patho)physiological processes. $\mathrm{Ca}^{2+}$ excitability $\mathrm{Ca}^{2+}$ excitability is a cellular property by which cells display intracellular $\mathrm{Ca}^{2+}$ changes that occur either spontaneously/intrinsically or in response to various stimuli, such as, touch and ligand.

\section{Micropatterning}

Micropatterning is an engineering approach of miniaturization to generate micrometer-sized patterns.
Similarly, electrical recordings from neurons revealed a role of nearby astrocytes in modulation of neuronal excitability, synaptic transmission, and plasticity (reviewed in Perea et al., 2009). However, the use of fluorescent $\mathrm{Ca}^{2+}$ indicators and dynamic imaging techniques demonstrated that astrocytes display an intracellular $\mathrm{Ca}^{2+}$ excitability (Cornell-Bell et al., 1990). Astrocytes can increase their intracellular $\mathrm{Ca}^{2+}$ levels in response to synaptic activity (Dani et al., 1992; Porter and McCarthy, 1996) and can, also, signal to neurons in a $\mathrm{Ca}^{2+}$-dependent manner (Nedergaard, 1994; Parpura et al., 1994). Hence, the combination of electrophysiology and $\mathrm{Ca}^{2+}$ imaging techniques allowed a new venue for investigations of the bidirectional signaling between neurons and astrocytes.

Because of the inherent complexity of the brain, studying its function often requires researchers to undertake various levels of analysis, ranging from systems, cell networks, single cells, down to the level of cellular compartments, such as, a tripartite synapse (see concept in Araque et al., 1999) and its molecular constituents. The advantage of characterizing neural processes in culture systems is the ability to control various experimental conditions in a highly defined manner, which has proven difficult in situ and in vivo preparations. Although such a reductionist approach yields some difference in observations between astrocytic properties in culture and in situ (Cahoy et al., 2008), general phenomena discovered in cell culture systems have also been found in vivo. For example, the discovery of $\mathrm{Ca}^{2+}$ excitability in astrocytes was first described in cell culture (Cornell-Bell et al., 1990) and since has also been demonstrated in brain slice preparations (Dani et al., 1992; Aguado et al., 2002; Nett et al., 2002) and in vivo (Hirase et al., 2004; Wang et al., 2006; Takata and Hirase, 2008). While recent advancements in imaging techniques allow for in vivo studies that have provided exciting new insights into the functional role of astrocytes in the intact healthy brain (reviewed in Nimmerjahn, 2009), there are still many issues in respect to astrocytic function which require addressing them with experiments using cell cultured astrocytes. For example, one such hot topic is the molecular identity of astrocytic "hemichannel": connexin 43 vs. pannexin 1 (Iglesias et al., 2009), where the use of culture cells allows controlled experimental conditions to record hemichannel activity. In this focused review, we discuss a subset of experiments in which micropatterned substrates were used to culture astrocytes in order to study their functions.

\section{MICROPATTERNED SUBSTRATES FOR ASTROCYTIC CELL CULTURE}

Micropatterning is an engineering approach of miniaturization to generate micrometersized patterns. Aerosol micropatterning is well adapted for biological materials. It uses spraying of the material where a suspension of fine liquid droplets in the air is dispersed in a semi-random pattern onto pre-treated glass coverslips. We illustrate the use of this technique in Section "Aerosol Micropatterning of Substrates: Microisland Culture Approach in Studying Bilateral Neuronal-Astrocytic Interactions", where, so called, microisland cultures were used to study bidirectional astrocyte-neuron signaling. An alternative technique used in neurosciences utilizes soft lithography, a standard procedure in microelectronics. Here, elastomeric molds are used to generate patterned features with well-defined and controllable spatial relationship. This approach generally uses photolithography to obtain molds, but also micromachining has been developed for this purpose. We illustrate these soft lithography approaches in Section "Soft Lithography Approach to Generate Micropatterned Substrates for Culturing Astrocytes".

\section{AEROSOL MICROPATTERNING OF SUBSTRATES: MICROISLAND CULTURE APPROACH IN STUDYING BILATERAL NEURONAL-ASTROCYTIC INTERACTIONS}

Microisland culturing approach was first used to characterize the chemical transmission between sympathetic neurons and cardiac myocytes (Furshpan et al., 1976). Since then, its use has been instrumental in probing interactions between neurons and astrocytes. The patterning of glass coverslips is done in two steps. First, coverslips are coated with a thin layer of agarose that prevents adherence of cells. Then, a cellgrowth permissive substrate, consisting of either collagen alone or its mixture with an organic polymer, such as, poly-D-lysine or polyethyleneimine, is sprayed onto the agarose layer forming randomly distributed microislands of various sizes. These patterned substrates can be used to plate mixed glial and neuronal cells (Figure 1A). Alternatively, astrocytes are initially plated onto patterned coverslips with these cells attaching to and occupying the majority of microislands. These glial cells serve as a feeder layer for neurons that are subsequently plated on top of them. In both approaches, single neurons grown on glial/ astrocytic microislands can form synapses onto itself, referred to as autapses. In this configuration the same neuron that is electrically stimulated to evoke transmitter release shows synaptic 


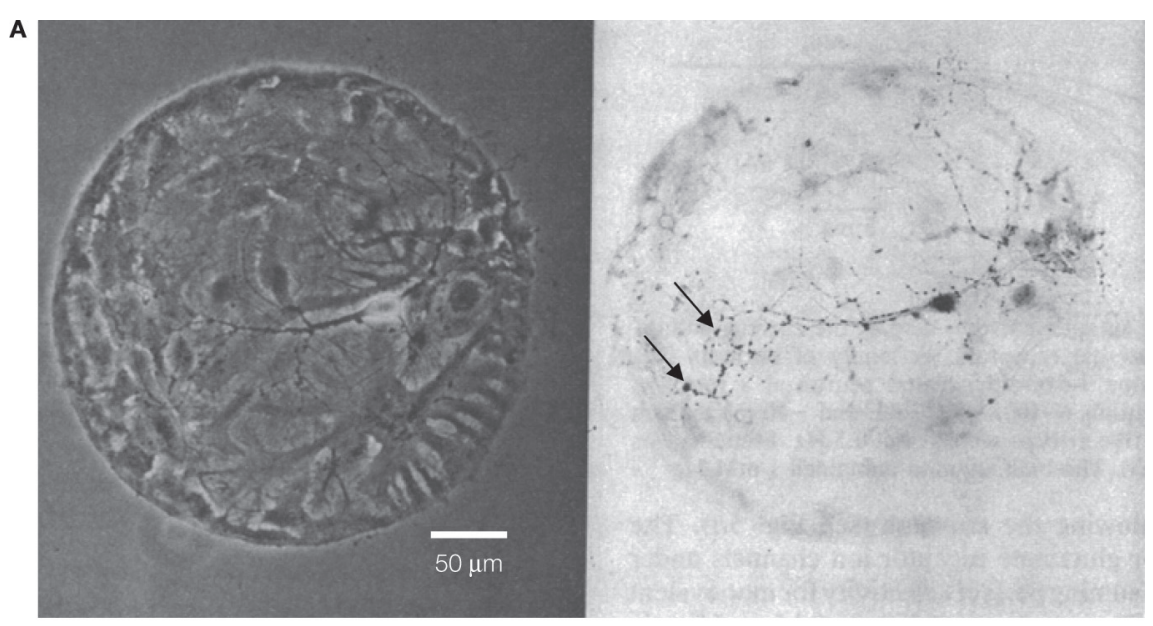

B
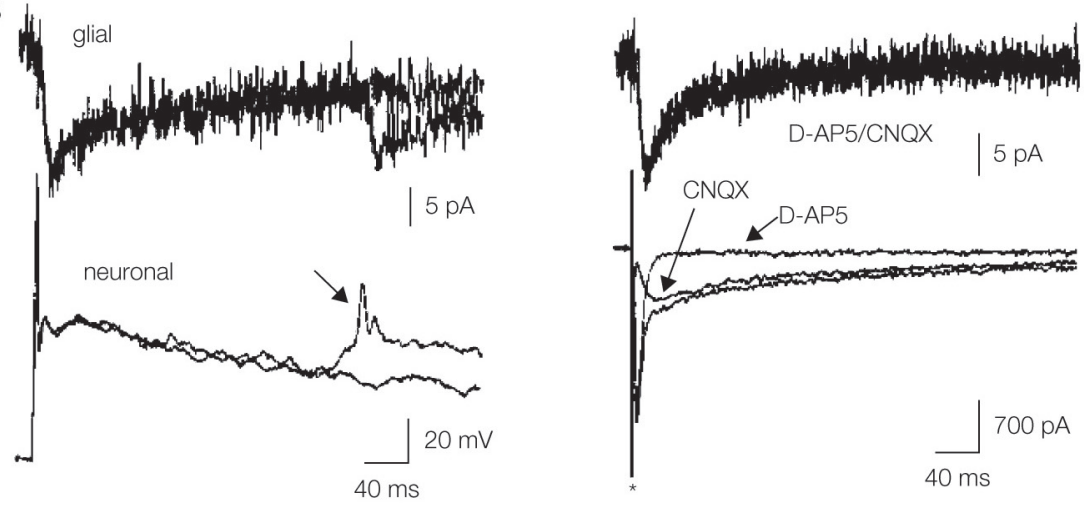

C
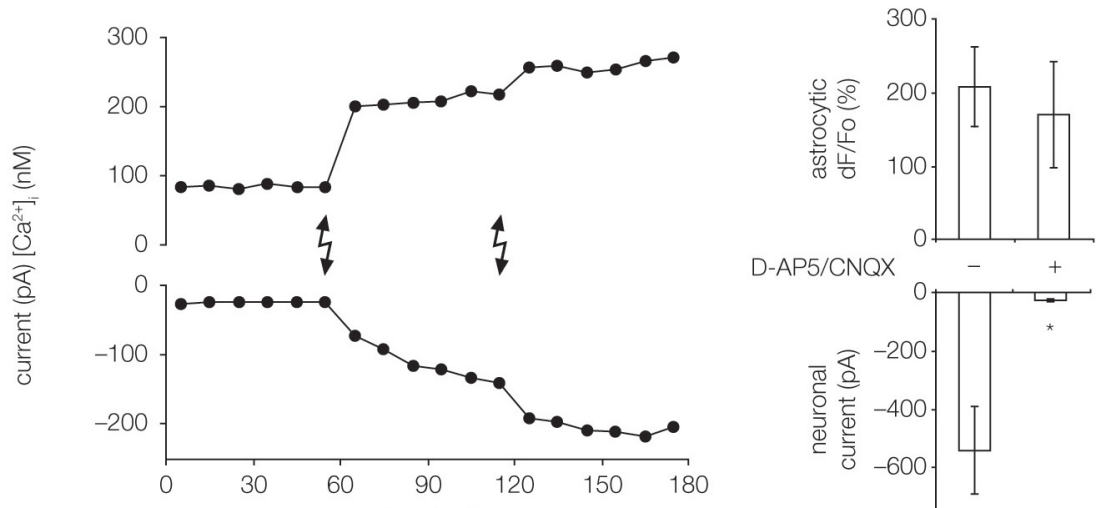

Figure 1 | Microisland cultures for studying neuronal-glial/astrocytic interactions. (A) Single neurons were grown on microislands consisting of glial cells, likely astrocytes (left, phase contrast). Cell permissive substrate, a mixture of collagen and poly-D-lysine, was aerosol micropatterned to form a spot onto an agarose coated glass coverslips. Hippocampal cell suspension was applied to dishes and cells adhered to permissive substrate forming microislands. Neurons were labeled (dark) with anti-synapsin I antibody (right, bright field); punctate staining (arrows) indicates putative autapses. Modified from Bekkers and Stevens (1991). (B) Neuron-to-glia signaling. Simultaneous electrical recordings obtained from a single hippocampal neuron (bottom) and astrocytes (top) residing on a microisland prepared as in (A). Glial cells (astrocytes) respond to synaptic transmission by an inward current (left), which represents an electrogenic activity of their plasma membrane glutamate transporters. Stimulation of a neuron to cause action potential evokes prolonged neuronal autaptic depolarization, which, in some cases, can drive an additional action potential (arrow) also causing a glial response. Ionotropic glutamate receptor antagonists (D-AP5 and CNOX) have no effect on astrocytic currents (right), while their use eliminated different components (slow and fast, respectively) of the autaptic currents in the voltage-clamped neuron. The asterisk indicates artifact due to application of a depolarizing step in the neuron. Modified from Mennerick and Zorumski (1994). (C) Astrocyte-to-neuron signaling. Neurons were plated onto pre-plated purified astrocytes forming microislands. Astrocytes occupying a microisland, loaded with the $\mathrm{Ca}^{2+}$ indicator and cage, were exposed to UV light (lighting bolts) to cause an increase in astrocytic [ $\left.\mathrm{Ca}^{2+}\right]$. Left: Simultaneous electrical recordings from a single neuron, grown on top of astrocytes, indicate that the physiological increase in astrocytic $\left[\mathrm{Ca}^{2+}\right]_{\text {; }}$ is sufficient to cause glutamate-mediated SICs in neurons. Right: D-AP5/CNQX significantly (asterisk) attenuated the ability of photolytic $\mathrm{Ca}^{2+}$ elevations in astrocytes [shown as dF/Fo (\%)] to cause neuronal SICs (right). Modified from Parpura and Haydon (2000). 
currents, thus, providing a minimal model for studying synaptic transmission (Bekkers and Stevens, 1991). Below, we discuss two examples of the use of microisland culturing technique to study bidirectional neuron-astrocyte signaling.

\section{Synaptic transmission affects the activity of astrocytic plasma membrane glutamate transporters}

Astrocytes are predominantly (over neurons) responsible for removing glutamate from synaptic clefts through the activity of high affinity glutamate transporters expressed on their plasma membranes (reviewed in Danbolt, 2001). This action maintains the extracellular glutamate concentration at about $\sim 25 \mathrm{nM}$ (Herman and Jahr, 2007). To assess the contribution of astrocytic glutamate transporters to synaptic transmission, Mennerick and Zorumski (1994), using microisland cultures, simultaneously monitored the electrical activity of single hippocampal neurons and the astrocytes underneath. Stimulation of a neuron elicited an autaptic event, recorded as either excitatory post-synaptic potentials or currents (Figure 1B). Post-synaptic currents contained dual activation of $n$-methyl-D-aspartate (NMDA) and non-NMDA ionotropic glutamate receptors, since respective receptor type antagonists, D-2-amino-5-phosphonopentanoic acid (D-AP5) or 6-cyano-7-nitroquinoxaline2,3-dione (CNQX), reduced their magnitude and eliminated their specific components. The autaptic transmission caused inward currents in the underlying astrocytes (Figure 1B, left). Such astrocytic currents were generated due to electrogenic activity of their plasma membrane glutamate transporters, since currents were unaltered in the presence of D-AP5/CNQX (Figure 1B, right), but they were sensitive to glutamate uptake inhibitors threo-3-hydroxyaspartate, trans-pyrrolidine-2,4-dicarboxylic acid, and lithium ions. The inhibition of astrocytic uptake of glutamate prolonged excitatory post-synaptic currents. Therefore, neurons can signal to astrocytes by synaptically released glutamate, which activates astrocytic glutamate uptake systems. In turn, astrocytic uptake of glutamate from extracellular space modulates the time-course of synaptic neurotransmission.

\section{Astrocytes release glutamate in $\mathrm{Ca}^{2+}$-dependent manner to signal to adjacent neurons}

Astrocytes display intracellular $\mathrm{Ca}^{2+}$ excitability (Cornell-Bell et al., 1990) which can lead to $\mathrm{Ca}^{2+}$ dependent glutamate release from these cells (Parpura et al., 1994). Intracellular elevations of
$\mathrm{Ca}^{2+}$ are sufficient and necessary to cause release of this gliotransmitter via regulated exocytosis (reviewed in Montana et al., 2006); for a review of other mechanism for glutamate release from astrocytes see Malarkey and Parpura (2008). In turn, glutamate released from astrocytes can have various effects in neurons including a slow inward current (SIC) (reviewed in Ni et al., 2007). The release of glutamate from astrocytes and the consequent signaling to neurons represents a physiological signaling pathway, as assessed using microisland cultures (Parpura and Haydon, 2000). Astrocytes and neurons were co-loaded with cell permeant forms of $\mathrm{Ca}^{2+}$ indicator and $\mathrm{Ca}^{2+}$ cage. Following the loading procedure, implementation of a simple dialysis protocol insured the absence of indicator/cage in the neuron. Since astrocytes underneath the dialyzed neuron still contained $\mathrm{Ca}^{2+}$ cage, its photolysis, caused by shining UV light onto cells, led to selective stimulation of astrocytes that increased their intracellular $\mathrm{Ca}^{2+}$ concentration $\left[\mathrm{Ca}^{2+}\right]_{i}$ and caused the neuronal SIC (Figure 1C, left). These SICs were mediated by the activation of NMDA and non-NMDA ionotropic glutamate receptors since amplitudes of photolytically induced SICs were greatly reduced when the microisland cultures were treated with the combination of D-AP5 and CNQX (Figure 1C, right). Taken together, $\mathrm{Ca}^{2+}$-dependent glutamate release from astrocytes operates within the physiological $\left[\mathrm{Ca}^{2+}\right]_{\mathrm{i}}$ levels seen in astrocytes, suggesting that it constitutes a relevant signaling pathway that can be used for astrocyte-neuron signaling in the CNS.

\section{SOFT LITHOGRAPHY APPROACH TO GENERATE MICROPATTERNED SUBSTRATES FOR CULTURING ASTROCYTES}

One of the drawbacks of aerosol micropatterning is the lack of control with respect to localization and size of cell-growth permissive areas. This can be circumvented by using soft lithography approach. Here, glass coverslips (or plastic dishes) pre-coated with a permissive substrate, such as poly-L-lysine or polyethyleneimine, are brought into contact with a mold made of an elastomer, usually poly(dimethylsiloxane) (PDMS). The mold design allows a deposition of agarose to coverslips while protecting some areas of pre-coated substrate upon which cells would attached at a later stage. After the agarose is set, the mold is removed from the glass coverslip, resulting in micropatterned substrate. PDMS mold is pre-casted by pouring an elastomer mixture onto a master template, followed by curing the mixture. Once cured, the 
Intercellular $\mathrm{Ca}^{2+}$ wave Intercellular $\mathrm{Ca}^{2+}$ wave is a subtype of $\mathrm{Ca}^{2+}$ excitability where increase in the intracellular $\mathrm{Ca}^{2+}$ of an individual cell spreads onto adjacent cells in the form of a wave. It may represent a type of long-range signaling amongst cells.
PDMS mold is peeled away from the template and is, then, ready for patterning coverslips. PDMS molds, as well as master templates, can be used multiple times. The master template can be generated using photolithography, an approach, which is used for studying a spread of elevated intracellular $\mathrm{Ca}^{2+}$ among astrocytes in the form of an intercellular $\mathrm{Ca}^{2+}$ wave (ICW) as illustrated in Section "Astrocyte-astrocyte $\mathrm{Ca}^{2+}$ dynamics: ATP-mediated intercellular $\mathrm{Ca}^{2+}$ waves (ICW)". Alternatively, a master template can be micromachined from a durable material, such as Teflon. The use of such an approach for studying astrocytic intracellular $\mathrm{Ca}^{2+}$ oscillations is illustrated in Section "Characteristics of astrocytic intracellular $\mathrm{Ca}^{2+}$ oscillations are affected by interactions among astrocytes".

\section{Astrocyte-astrocyte $\mathrm{Ca}^{2+}$ dynamics: ATP-mediated intercellular $\mathrm{Ca}^{2+}$ waves (ICW)}

Cornell-Bell et al. (1990) made the seminal observation that $\mathrm{Ca}^{2+}$ excitability of individual cultured hippocampal astrocytes can spread onto adjacent cells in the form of ICW. With increased astrocytic $\mathrm{Ca}^{2+}$ excitability, which can be induced e.g., by mechanical stimulation, adenosine $5^{\prime}$-triphosphate (ATP) can be released from astrocytes into extracellular space (Coco et al., 2003). In turn, ATP activation of various purinergic receptors can lead to an increase of $\left[\mathrm{Ca}^{2+}\right]_{\mathrm{i}}$ in neighboring astrocytes. This diffusion of ATP through the extracellular space appears to be the primary mechanism of the propagation of ICWs (Guthrie et al., 1999; Cotrina et al., 2000; Arcuino et al., 2002; Stout et al., 2002), although gap junctions also contribute to this process (Venance et al., 1997). One approach to assess the contributions of these two components, diffusion factor vs. gap junctions, is to test whether ICWs can propagate between cells that are physically separated. Takano et al. (2002) conducted such experiments using micropatterned coverslips containing alternating stripes of poly-L-lysine and agarose (Figure 2A). Astrocytes plated onto patterned coverslips adhered to poly-L-lysine stripes, while agarose stripes were devoid of cellular components. When astrocytes loaded with a $\mathrm{Ca}^{2+}$ indicator were mechanically stimulated, there was an increase of $\left[\mathrm{Ca}^{2+}\right]_{i}$ which propagated as an ICW, not only throughout the primary stripe where the mechanical stimulation was applied, but also via physically separated nearby stripes (Figure 2B). This spread of ICWs within the primary stripe and between adjacent stripes was greatly reduced in the presence of a purinergic receptor blocker. Thus, this study confirms ATP as a molecule being released from astrocytes during times of $\mathrm{Ca}^{2+}$ excitability and then diffusing through the extracellular space in support of propagating ICWs among these glial cells.

\section{Characteristics of astrocytic intracellular $\mathrm{Ca}^{2+}$ oscillations are affected by interactions among astrocytes}

Cornell-Bell et al. (1990) used cultured and confluent astrocytes to demonstrate intracellular $\mathrm{Ca}^{2+}$ excitability. Astrocytes displayed a variety of distinct patterns of increased $\left[\mathrm{Ca}^{2+}\right]_{i}$, either sustained oscillations with constant or decreasing frequencies, or damped-oscillations and step responses. Consequently, a notion that $\left[\mathrm{Ca}^{2+}\right]_{i}$ changes, especially frequency of oscillations, can be used for encoding information transfer by astrocytes was put forth (Pasti et al., 1997), although the concept still remains speculative. Interestingly, solitary astrocytes devoid of contact can also exhibit $\left[\mathrm{Ca}^{2+}\right]_{i}$ oscillations (Hua et al., 2004). To systematically study whether astrocytic $\left[\mathrm{Ca}^{2+}\right]_{i}$ oscillation patterns depends on cell-cell interactions, Lee et al. (2008) developed a micropit cell culturing method. Here, circular micropits, in which the bottoms were coated with polyethyleneimine, were used to control the localization of solitary astrocytes or small groups of these cells. An additional feature of this culturing method is that on the same coverslip containing micropits, there were also two patterned large segmental (non-pit) regions used for common culturing of confluent astrocytes (Figure 2C); cell permissive pit/ non-pit regions were surrounded by agarose. Solitary astrocytes that occupied a subset of micropits exhibited similar characteristics in terms of astrocytic marker expression to those of astrocytes grown in groups in a confluent monolayer in non-pit regions. However, solitary micropit astrocytes displayed less variability of $\mathrm{Ca}^{2+}$ oscillations when compared to $\mathrm{Ca}^{2+}$ dynamics recorded from grouped astrocytes in non-pit regions (Figure $2 \mathrm{D}$ ). Thus, the complexity of astrocytic intracellular $\mathrm{Ca}^{2+}$ oscillatory pattern appears to be due to the astrocytic network and interactions between neighboring cells.

\section{CONCLUDING REMARKS}

The purpose of this focused review is to discuss some of the uses of micropatterned substrates for cell culture systems in investigation of astrocytic properties and functions. We point out that while astrocytes in culture might not exhibit the exact same characteristics as those found in vivo, most of the underlying mechanisms and cellular processes governing 

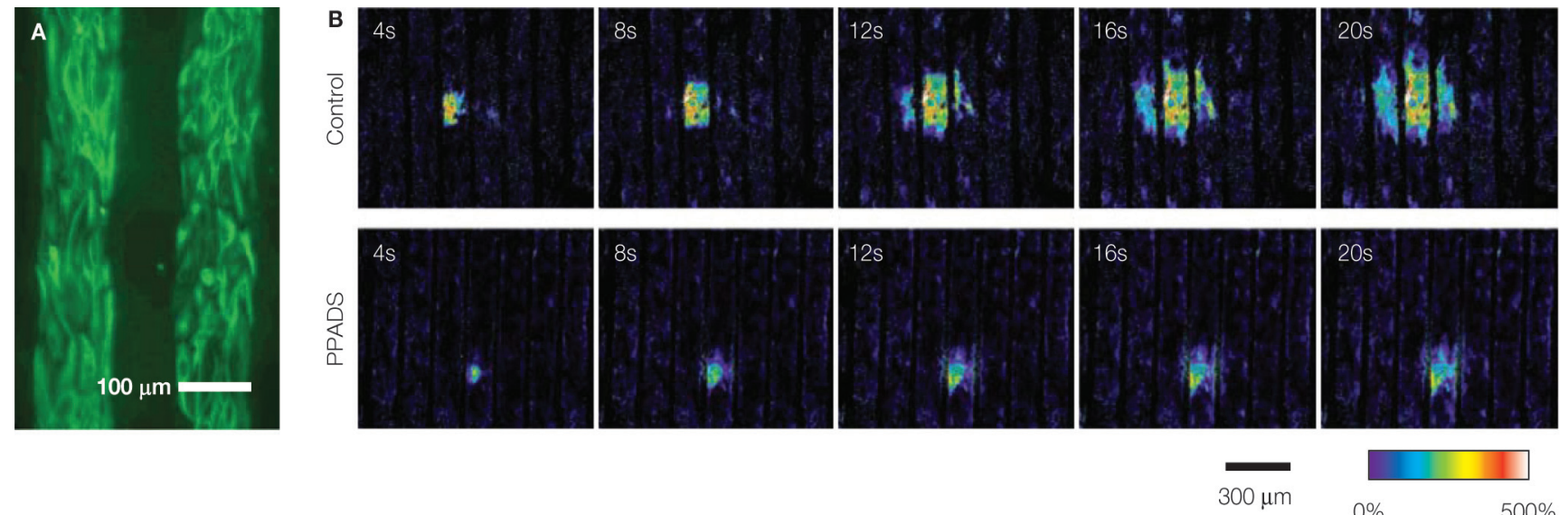

$0 \%$
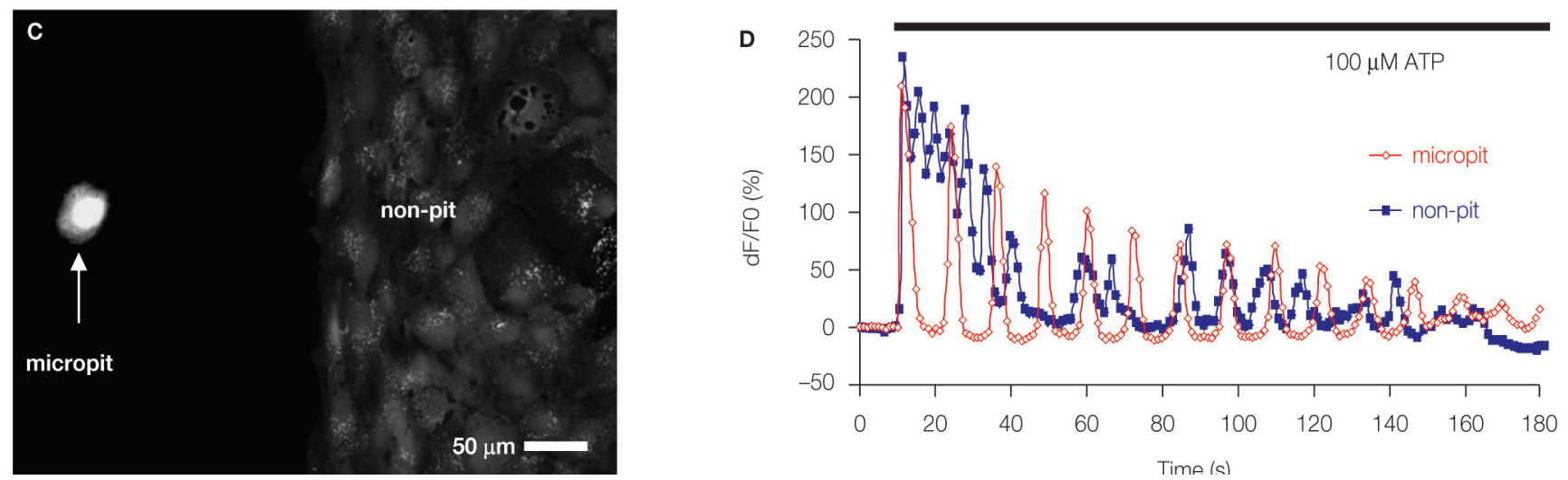

Figure 2 | Soft lithography micropatterned substrates and their use for investigation of astrocytic $\mathbf{C a}^{2+}$ dynamics. (A,B) ATP-mediated intercellular $\mathrm{Ca}^{2+}$ waves (ICW) among astrocytes. (A) Astrocytes grown on a glass coverslips patterned with alternating stripes of poly-L-lysine and agarose. Astrocytes adhered to the permissive substrate poly-L-lysine as indicated by labeling using indirect immunocytochemistry and an antibody against the astrocytic marker glial fibrillary acidic protein (GFAP; green). (B) Astrocytes cultured on striped micropatterned substrate [note that agarose stripes are narrower than in $(\mathbf{A})$ ] can be used to investigate ICW. Mechanical stimulation of astrocytes within the primary stripe (the third stripe from left) caused intracellular $\mathrm{Ca}^{2+}$ elevation that spread within the stripe and to the adjacent stripes in form of ICW (Control), implicating an involvement of a diffusible factor, which is also consistent with the circular pattern of ICW. The main diffusible molecule released from stimulated astrocytes, which supports the spread of ICW, is ATP, since the purinergic receptor antagonist pyridoxal-phosphate-6-azophenyl-2', $4^{\prime}$-disulfonate (PPADS) in extracellular solution reduced the extent of ICW. Time elapsed from mechanical

stimulation is indicated on individual images in seconds. $\mathrm{Ca}^{2+}$ dynamics were visualized using a $\mathrm{Ca}^{2+}$ indicator, which fluorescence changes are shown as dF/Fo (\%) in pseudocolor. Modified from Takano et al. (2002). (C,D) Characteristics of astrocytic intracellular $\mathrm{Ca}^{2+}$ oscillations are affected by interactions among astrocytes. (C) A micropatterned coverslip that contains cell-adhesive substrate polyethyleneimine in a micropit (left) and a non-pit region (right) surrounded by agarose (dark). Solitary astrocytes can populate the micropit regions, while non-pit regions contain confluent astrocytes, as evident from labeling with the vital stain calcein. (D) Time-lapse sequence of astrocytic intracellular $\mathrm{Ca}^{2+}$ changes in response to stimulation with ATP (horizontal bar). The $\left[\mathrm{Ca}^{2+}\right]$. oscillations from a solitary astrocyte in the micropit show regular inter-peak intervals and dampening in amplitude (red trace).

The intracellular $\mathrm{Ca}^{2+}$ response of a single cell within the group of confluent astrocytes in the non-micropit region displays an oscillatory pattern with various inter-peak intervals. The graphs represent a $\mathrm{Ca}^{2+}$ indicator fluorescence changes expressed as dF/Fo (\%). Modified from Lee et al. (2008)

astrocytic $\mathrm{Ca}^{2+}$ excitability and bidirectional astrocyte-neuron signaling in vitro have been subsequently found in brain slices and/or in vivo (reviewed in $\mathrm{Ni}$ et al., 2007; see the notable exception in Fiacco et al., 2007). It is important, however, to understand the limitations of a particular cell culture system used. Nonetheless, the power of astrocytic cell culturing methods lies in the control over many experimental variables to study the phenomenon of interest. The information yielded from using this experimental approach has spurred some novel ideas and models that were then tested in more intact systems, including in vivo. 


\section{ACKNOWLEDGMENTS}

We thank Reno C. Reyes and J. Robert Grammer for their comments on previous versions of this manuscript. The authors' work is supported by a grant from the National Institute of Mental Health (MH 069791 to V.P.). We dedicate this focused review to the late Glenn I. Hatton, whose work inspired new views of astrocyte-neuronal interactions.

\section{REFERENCES}

Aguado, F., Espinosa-Parrilla, J. F., Carmona, M. A., and Soriano, E. (2002). Neuronal activity regulates correlated network properties of spontaneous calcium transients in astrocytes in situ. J. Neurosci. 22, 9430-9444.

Araque, A., Parpura, V., Sanzgiri, R. P., and Haydon, P. G. (1999). Tripartite synapses: Glia, the unacknowledged partner. Trends Neurosci. 22, 208-215.

Arcuino, G., Lin, J. H., Takano, T., Liu, C., Jiang, L., Gao, Q., Kang, J., and Nedergaard, M. (2002). Intercellular calcium signaling mediated by pointsource burst release of ATP. Proc. Natl. Acad. Sci. U.S.A. 99, 9840-9845.

Bekkers, J. M., and Stevens, C. F. (1991). Excitatory and inhibitory autaptic currents in isolated hippocampal neurons maintained in cell culture. Proc. Natl. Acad. Sci. U.S.A. 88, 7834-7838.

Binder, D. K., and Steinhäuser, C. (2009). Role of astrocytes in epilepsy. In Astrocytes in (patho)physiology of the nervous system, V. Parpura and P. G. Haydon, eds (Boston, MA, Springer), pp. 649-671.

Brenner,M.,Goldman,J.E., Quinlan,R. A., and Messing, A. (2009). Alexander disease: a genetic disorder of astrocytes. In Astrocytes in (patho)physiology of the nervous system, V. Parpura and P. G. Haydon, eds (Boston, MA, Springer), pp. 591-648.

Butterworth, R. F. (2009). Hepatic encephalopathy: a primary astrocytopathy. In Astrocytes in (patho)physiology of the nervous system, V. Parpura and P. G. Haydon, eds (Boston, MA: Springer), pp. 673-692.

Cahoy, J. D., Emery, B., Kaushal, A., Foo, L. C., Zamanian, J. L., Christopherson, K. S., Xing, Y., Lubischer, J. L., Krieg, P. A., Krupenko, S.A., Thompson, W.J., and Barres, B. A. (2008). A transcriptome database for astrocytes, neurons, and oligodendrocytes: a new resource for understanding brain development and function. J. Neurosci. 28, 264-278.

Coco, S., Calegari, F., Pravettoni, E., Pozzi, D., Taverna, E., Rosa, P., Matteoli, M., and Verderio, C. (2003). Storage and release of ATP from astrocytes in culture. J. Biol. Chem. 278, 1354-1362.

Cornell-Bell, A. H., Finkbeiner, S. M., Cooper, M. S., and Smith, S. J. (1990). Glutamate induces calcium waves in cultured astrocytes: long-range glial signaling. Science 247, 470-473.

Cotrina,M.L.,Lin,J.H.,Lopez-Garcia, J. C., Naus, C. C., and Nedergaard, M. (2000). ATP-mediated glia signaling. J. Neurosci. 20, 2835-2844.

Danbolt, N.C. (2001). Glutamate uptake. Prog. Neurobiol. 65, 1-105.

Dani,J.W.,Chernjavsky,A., and Smith, S. J. (1992). Neuronal activity triggers calcium waves in hippocampal astrocyte networks. Neuron 8, 429-440.

Fiacco, T. A., Agulhonm, C., Taves, S. R., Petravicz, J., Casper, K. B., Dong, X., Chen, J.,. and McCarthy, K. D. (2007) Selective stimulation of astrocyte calcium in situ does not affect neuronal excitatory synaptic activity. Neuron 54, 611-626.

Furshpan, E. J., MacLeish, P. R., O'Lague, P. H., and Potter, D. D. (1976). Chemical transmission between rat sympathetic neurons and cardiac myocytes developing in microcultures: Evidence for cholinergic, adrenergic, and dual-function neurons. Proc. Natl. Acad. Sci. U.S.A. 73, 4225-4229.

Gordon, G. R. J., Mulligan, S. J., and MacVicar, B. A. (2009). Astrocyte control of blood flow. In Astrocytes in (patho)physiology of the nervous system, V. Parpura and P. G. Haydon, eds (Boston, MA, Springer), pp. 461-486.

Guthrie, P. B., Knappenberger, J., Segal, M., Bennett, M. V., Charles, A. C., and Kater, S. B. (1999). ATP released from astrocytes mediates glial calcium waves. J. Neurosci. 19, 520-528.

Herman, M. A., and Jahr, C. E. (2007). Extracellular glutamate concentration in hippocampal slice. J. Neurosci. 27, 9736-9741.

Hirase, H., Qian, L., Bartho, P., and Buzsaki, G. (2004). Calcium dynamics of cortical astrocytic networks in vivo. PLoS Biol. 2, E96. doi: 10.1371/ journal.pbio.0020096.

Hua, X., Malarkey, E. B., Sunjara, V., Rosenwald, S. E., Li, W. H., and Parpura, V. (2004). $\mathrm{Ca}^{2+}$-dependent glutamate release involves two classes of endoplasmic reticulum $\mathrm{Ca}^{2+}$ stores in astrocytes. J. Neurosci. Res. 76, 86-97.

Iglesias, R., Dahl, G., Qiu, F., Spray, D. C., and Scemes, E. (2009). Pannexin 1: The molecular substrate of astrocyte "hemichannels". J. Neurosci. 29, 7092-7097.
Kuchibhotla, K. V., Lattarulo, C. R., Hyman, B. T., and Bacskai, B. J. (2009). Synchronous hyperactivity and intercellular calcium waves in astrocytes in alzheimer mice. Science 323, 1211-1215.

Lee, W., Malarkey, E. B., Reyes, R. C., and Parpura, V. (2008). Micropit: a new cell culturing approach for characterization of solitary astrocytes and smal networks of these glial cells. Front Neuroengineering 1, 2. doi: 10.3389/ neuro.16.002.2008.

Malarkey, E. B., and Parpura, V. (2008) Mechanisms of glutamate release from astrocytes. Neurochem. Int. 52, 142-154.

Mennerick, S., and Zorumski, C. F. (1994). Glial contributions to excitatory neurotransmission in cultured hippocampal cells. Nature 368, 59-62.

Montana, V., Malarkey, E. B., Verderio, C. Matteoli, M., and Parpura, V. (2006). Vesicular transmitter release from astrocytes. Glia 54, 700-715.

Nedergaard, M. (1994). Direct signaling from astrocytes to neurons in cultures of mammalian brain cells. Science 263 1768-1771.

Nett,W.J.,Oloff,S.H., and McCarthy, K. D. (2002). Hippocampal astrocytes in situ exhibit calcium oscillations that occur independent of neuronal activity. J. Neurophysiol. 87, 528-537.

Ni, Y., Malarkey, E. B., and Parpura, V. (2007). Vesicular release of glutamate mediates bidirectional signaling between astrocytes and neurons. J. Neurochem. 103, 1273-1284.

Nimmerjahn, A. (2009). Astrocytes going live: Advances and challenges. J. Physiol. 587, 1639-1647.

Parpura, V., and Haydon, P. G. (2000) Physiological astrocytic calcium levels stimulate glutamate release to modulate adjacent neurons. Proc. Natl. Acad. Sci. U.S.A. 97, 8629-8634.

Parpura, V., Basarsky, T. A., Liu, F., Jeftinija, K., Jeftinija, S., and Haydon, P. G. (1994). Glutamatemediated astrocyte-neuron signalling. Nature 369, 744-747.

Pasti, L., Volterra, A., Pozzan, T., and Carmignoto, G. (1997).Intracellular calcium oscillations in astrocytes: a highly plastic, bidirectional form of communication between neurons and astrocytes in situ. J. Neurosci. 17, 7817-7830.

Perea, G., Navarrete, M., and Araque, A. (2009). Tripartite synapses: Astrocytes processand controlsynapticinformation. Trends Neurosci. 32, 421-431.
Porter, J. T., and McCarthy, K. D. (1996). Hippocampal astrocytes in situ respond to glutamate released from synaptic terminals. J. Neurosci. 16, 5073-5081.

Stout, C. E., Costantin, J. L., Naus, C. C., and Charles, A.C. (2002). Intercellular calcium signaling in astrocytes via ATP release through connexin hemichannels. J. Biol. Chem. 277, 10482-10488.

Takano, H., Sul, J. Y., Mazzanti, M. L., Doyle, R. T., Haydon, P. G., and Porter, M. D. (2002). Micropatterned substrates: Approach to probing intercellular communication pathways. Anal. Chem. 74, 4640-4646.

Takata, N., and Hirase, H. (2008). Cortical layer 1 and layer $2 / 3$ astrocytes exhibit distinct calcium dynamics in vivo. PLoS One 3, e2525. doi: 10.1371/ journal.pone.0002525

Tzingounis, A.V., and Wadiche, J. I. (2007). Glutamate transporters: Confining runaway excitation by shaping synaptic transmission. Nat. Rev. Neurosci. 8, 935-947.

Venance, L., Stella, N., Glowinski, J. and Giaume, C. (1997). Mechanism involved in initiation and propagation of receptor-induced intercellular calcium signaling in cultured rat astrocytes. J. Neurosci. 17, 1981-1992.

Wang, X., Lou, N., Xu, Q., Tian, G. F., Peng, W. G., Han, X., Kang, J., Takano, T., and Nedergaard, M. (2006). Astrocytic $\mathrm{Ca}^{2+}$ signaling evoked by sensory stimulation in vivo. Nat. Neurosci. 9, 816-823.

Conflict of Interest Statement: The authors declare that the research was conducted in the absence of any commercial or financial relationships that could be construed as a potential conflict of interest.

Received: 24 July 2009; paper pending published: 28 August 2009; accepted: 03 September 2009; published: 15 December 2009.

Citation: Front. Neurosci. (2009) 3, 1:381387. doi: 10.3389/neuro.01.033.2009

Copyright $\odot 2009$ Lee and Parpura. This is an open-access publication subject to an exclusive license agreement between the authors and the Frontiers Research Foundation, which permits unrestricted use, distribution, and reproduction in any medium, provided the original authors and source are credited. 